\title{
Bifurcation and exact solutions for the $(2+1)$-dimensional conformable time-fractional Zoomeron equation
}

Zhao Li ${ }^{1 *}$ (D) and Tianyong Han ${ }^{1}$

Correspondence:

lizhao10.26@163.com

${ }^{1}$ College of Computer Science, Chengdu University, Chengdu, China

\section{를 Springer}

\begin{abstract}
In this paper, the bifurcation and new exact solutions for the $(2+1)$-dimensional conformable time-fractional Zoomeron equation are investigated by utilizing two reliable methods, which are generalized $\left(G^{\prime} / G\right)$-expansion method and the integral bifurcation method. The exact solutions of the $(2+1)$-dimensional conformable time-fractional Zoomeron equation are obtained by utilizing the generalized $\left(G^{\prime} / G\right)$-expansion method, these solutions are classified as hyperbolic function solutions, trigonometric function solutions, and rational function solutions. Giving different parameter conditions, many integral bifurcations, phase portraits, and traveling wave solutions for the equation are obtained via the integral bifurcation method. Graphical representations of different kinds of the exact solutions reveal that the two methods are of significance for constructing the exact solutions of fractional partial differential equation.
\end{abstract}

Keywords: Bifurcation; $\left(G^{\prime} / G\right)$-expansion; Exact solution; Conformable fractional derivative; $(2+1)$-dimensional conformable time-fractional Zoomeron equation

\section{Introduction}

It is well known that the fractional partial differential equations (FPDEs) [1-11] have received considerable attention [12-19] due to their wide use to describe various complex physical phenomena in the domain of science and engineering. Among the research of the FPDEs, analyzing the bifurcations and the exact traveling wave solutions of FPDEs have been widely investigated as an important subject. Recently, many effective methods have been established and developed to analyze the dynamical behavior of the FPDEs. These methods include the $\left(G^{\prime} / G\right)$-expansion method, the integral bifurcations, the Lie symmetry analysis method, the exp-function method, the Kudryashov method, and so on.

It is worth noting that the $\left(G^{\prime} / G\right)$-expansion method, which was first introduced in [20], has made significant achievements in searching for the exact traveling wave solutions of partial differential equations (PDEs). But the exact solutions of FPDEs have been developed very slowly compared to the exact traveling solutions of PDEs. Most of the methods directly transform an FPDE into an ordinary differential equation by a fractional complex transformation. But the Jumarie's fractional chain rule does not hold. Therefore, the

(c) The Author(s) 2020. This article is licensed under a Creative Commons Attribution 4.0 International License, which permits use, sharing, adaptation, distribution and reproduction in any medium or format, as long as you give appropriate credit to the original author(s) and the source, provide a link to the Creative Commons licence, and indicate if changes were made. The images or other third party material in this article are included in the article's Creative Commons licence, unless indicated otherwise in a credit line to the material. If material is not included in the article's Creative Commons licence and your intended use is not permitted by statutory regulation or exceeds the permitted use, you will need to obtain permission directly from the copyright holder. To view a copy of this licence, visit http://creativecommons.org/licenses/by/4.0/ 
fractional complex transformation cannot be used to obtain the exact traveling solutions of FPDEs when the Riemann-Liouville derivative is used. Recently, Khalil and coworkers [21] introduced the conformable fractional derivative. After that, some scholars [22-25] have begun to discuss the exact solutions of FPDEs in the sense of the conformable fractional derivative. In this paper, we will introduce the procedure of the generalized $\left(G^{\prime} / G\right)$ expansion method for FPDEs, and will discuss the exact traveling wave solutions of the $(2+1)$-dimensional conformable time-fractional Zoomeron equation by the generalized $\left(G^{\prime} / G\right)$-expansion method together with conformable fractional derivative.

The bifurcation method first proposed by Liu and Li [26] is one of the most powerful tools to study the dynamic behavior of PDEs, especially in the analysis of the bifurcation and exact traveling wave solutions [27-30]. As far as we know, the bifurcation method has not been used to investigate the exact traveling wave solutions of FPDEs in the sense of the conformable fractional derivative. In the paper, we will introduce the procedure of bifurcation approach for constructing the exact traveling wave solutions of FPDEs. By using this method, we will analyze the bifurcation and exact solutions of the $(2+1)$-dimensional conformable time-fractional Zoomeron equation.

The Zoomeron equation is a very convenient model which displays the novel phenomena related with boomerons and trappons, this equation is usually used to describe the evolution of a single scalar field. Recently, Odabasi [31] studied the following $(2+1)$ dimensional conformable time-fractional Zoomeron equation:

$$
\frac{\partial^{2 \alpha} u}{\partial t^{2 \alpha}}\left[\frac{u_{x y}}{u}\right]-\frac{\partial^{2} u}{\partial x^{2}}\left[\frac{u_{x y}}{u}\right]+2 \frac{\partial^{\alpha} u}{\partial t^{\alpha}}\left[u^{2}\right]_{x}=0, \quad 0<\alpha \leq 1
$$

where $\frac{\partial^{\alpha} u}{\partial t^{\alpha}}$ is the conformable fractional derivative of $u$ depending on the variable $t$. Odabasi applied the modified trial equation method to obtain the exact solutions of the $(2+1)$-dimensional conformable time-fractional Zoomeron equation. Kumar and Kaplan [32] applied the extended $\exp (-\Phi(\xi))$-expansion technique and the exponential rational functional technique to find the explicit and exact solutions of the $(2+1)$-dimensional conformable time-fractional Zoomeron equation. Hosseini et al. [33] adopted the exp $(-\Phi(\xi))$ expansion approach and modified Kudryashov method to search for the exact solutions of the $(2+1)$-dimensional conformable time-fractional Zoomeron equation.

The main objective of the paper is to employ the generalized $\left(G^{\prime} / G\right)$-expansion method and bifurcation method to construct exact traveling wave solutions of the $(2+1)$ dimensional conformable time-fractional Zoomeron equation. The remainder of the article is structured as follows: In Sect. 2, we review the definition of the conformable fractional derivative, and introduce two effective methods for constructing the exact traveling wave solutions of FPDEs. Then in Sect. 3, we discuss the exact solutions of the $(2+1)$ dimensional conformable time-fractional Zoomeron equation by using the generalized $\left(G^{\prime} / G\right)$-expansion method and bifurcation method, respectively. Moreover, we obtain the bifurcation and phase portraits of this equation. Finally, we give a brief conclusion in Sect. 4.

\section{Mathematical preliminaries}

\subsection{The conformable fractional derivative}

The definition of the conformable fractional derivative is defined as in [34]. 
Definition 2.1 Let $f:[0, \infty) \rightarrow \mathbf{R}$. Then, the conformable fractional derivative of $f$ of order $\alpha$ is defined as

$$
D_{t}^{\alpha} f(t)=\lim _{\varepsilon \rightarrow 0} \frac{f\left(t+\varepsilon t^{1-\alpha}\right)-f(t)}{\varepsilon}, \quad \forall t \in(0,+\infty), \alpha \in(0,1],
$$

the function $f$ is $\alpha$-conformably differentiable at a point $t$ if the limit in equation (2.1) exists.

Remark 2.1 The conformable fractional derivative possesses the following properties:

(i) $D_{t}^{\alpha}\left(t^{\mu}\right)=\mu t^{\mu-\alpha}, \forall \mu \in \mathbf{R}$.

(ii) $D_{t}^{\alpha}(a f(t)+b g(t))=a D_{t}^{\alpha} f(t)+b D_{t}^{\alpha} g(t), \forall a, b \in \mathbf{R}$.

(iii) $D_{t}^{\alpha}(f \circ g)(t)=\left.t^{1-\alpha} g(t)^{\alpha-1} g^{\prime}(t) D_{t}^{\alpha}(f(t))\right|_{t=g(t)}$.

The conformable fractional derivative has many important properties. The detailed proof is given in the Appendix.

\subsection{Description of the methods}

Consider the following conformable FPDE:

$$
\mathrm{F}\left(u, \frac{\partial^{\alpha} u}{\partial t^{\alpha}}, \frac{\partial u}{\partial x}, \frac{\partial u}{\partial y}, \frac{\partial^{2 \alpha} u}{\partial t^{2 \alpha}}, \frac{\partial^{2} u}{\partial x^{2}}, \frac{\partial^{2} u}{\partial y^{2}}, \ldots\right)=0, \quad 0<\alpha \leq 1,
$$

where $t, x, y \in \mathrm{R}, u=u(t, x, y) \in \mathrm{R}, \mathrm{F}$ is a polynomial in $u$ and its partial fractional-order derivatives.

Introduce a traveling wave transformation

$$
u(t, x)=u(\xi), \quad \xi=k x+m y-\frac{l t^{\alpha}}{\alpha},
$$

where $k, m$ and $l$ are arbitrary constants.

Equation (2.2) is reduced to the following integer-order ordinary differential equation:

$$
\mathrm{P}\left(u, u^{\prime}, u^{\prime \prime}, \ldots\right)=0 \text {, }
$$

where $\mathrm{P}$ is a polynomial in $u$ and its derivatives, notation (') means the derivative with respect to $\xi$. If it is possible, we should integrate several times equation (2.4) and take the integral constants as zero.

\subsubsection{The generalized $\left(G^{\prime} / G\right)$-expansion method}

Step 1. Assume that the solution of equation (2.4) can be expressed as

$$
u(\xi)=\sum_{i=0}^{N} a_{i}\left(d+\frac{G^{\prime}}{G}\right)^{i}+\sum_{i=1}^{N} b_{i}\left(d+\frac{G^{\prime}}{G}\right)^{-i},
$$

where $d$ is an arbitrary constant, while $a_{i}(i=0,1,2, \ldots, N)$ and $b_{i}(i=1,2, \ldots, N)$ are to be determined later. Then $G=G(\xi)$ satisfies the following nonlinear ordinary differential equation:

$$
A G G^{\prime \prime}=B G G^{\prime}+C\left(G^{\prime}\right)^{2}+D G^{2}
$$

where $A, B, C$, and $D$ are real parameters. 
Step 2 . The positive integer $N$ can be computed by balancing the highest order derivative and nonlinear term appearing in equation (2.4).

Step 3. Substituting equation (2.5) together with equation (2.6) into equation (2.4), we get polynomials in $\left(d+\frac{G^{\prime}}{G}\right)^{N}(N=0, \pm 1, \pm 2, \ldots)$. Setting all the coefficients of the resulting polynomial to zero, we obtain algebraic equations.

Step 4. By solving the algebraic equations obtained in Step 3, we can obtain the values of the constants $a_{i}(i=0,1,2, \ldots)$ and $b_{i}(i=1,2, \ldots, N)$. Replacing their values in equation (2.5), we construct the exact solutions of equation (2.2).

\subsubsection{The bifurcation method}

Step 1. Let $\frac{d u}{d \xi}=y$. Equation (2.4) can be transformed into the following two-dimensional system:

$$
\left\{\begin{array}{l}
\frac{d u}{d \xi}=y, \\
\frac{d y}{d \xi}=\mathrm{R}(u, y),
\end{array}\right.
$$

where $\mathrm{R}(u, y)$ is an integral expression.

Step 2. Solve system (2.7) is an integral system, which has the first integral

$$
\mathrm{H}(u, y)=h,
$$

where $h$ is an integral constant.

Step 3. By employing the first integral $\mathrm{H}(u, y)$ and analyzing the orbit properties in the phase plane, we can obtain the exact solutions of equation (2.2).

\section{Applications}

By employing the transformation $u(t, x, y)=u(\xi), \xi=k x+m y+\frac{l t^{\alpha}}{\alpha}$, the $(2+1)$-dimensional conformable time-fractional Zoomeron equation can be reduced to an ordinary differential equation having form

$$
k m l^{2}\left[\frac{u^{\prime \prime}}{u}\right]^{\prime \prime}-k^{3} m\left[\frac{u^{\prime \prime}}{u}\right]^{\prime \prime}-2 k l\left[u^{2}\right]^{\prime \prime}=0 .
$$

Integrating equation (3.1) twice with respect to $\xi$, we obtain

$$
k m\left(l^{2}-k^{2}\right) u^{\prime \prime}-2 k l u^{3}-\rho u=0,
$$

where $\rho$ is a constant of integration.

\subsection{Exact solutions of equation (1.1) using the generalized ( $\left.G^{\prime} / G\right)$-expansion method}

Balancing $u^{\prime \prime}$ and $u^{3}$ in equation (3.2), we obtain $N=1$. Therefore, the solution form of equation (3.2) is

$$
u(\xi)=a_{1}\left(d+\frac{G^{\prime}}{G}\right)+a_{0}+b_{1}\left(d+\frac{G^{\prime}}{G}\right)^{-1} .
$$


Substituting (3.3) into (3.2) yields a polynomial in $\left(d+\frac{G^{\prime}}{G}\right)^{N}(N=0,1,2,3)$ and $\left(d+\frac{G^{\prime}}{G}\right)^{-N}$ $(N=1,2,3)$. Collecting the coefficients of the resulting polynomial, we obtain a system of nonlinear algebraic equations:

$$
\begin{aligned}
& \left(d+\frac{G^{\prime}}{G}\right)^{3}: k m\left(l^{2}-k^{2}\right) \cdot \frac{2 a_{1}(C-A)^{2}}{A^{2}}-2 k l a_{1}^{3}=0 ; \\
& \left(d+\frac{G^{\prime}}{G}\right)^{2}: k m\left(l^{2}-k^{2}\right) \cdot \frac{3 a_{1}(C-A)[B-2 d(C-A)]}{A^{2}}-2 k l \cdot 3 a_{0} a_{1}^{2}=0 ; \\
& \left(d+\frac{G^{\prime}}{G}\right)^{1}: k m\left(l^{2}-k^{2}\right)\left\{\frac{2 a_{1}(C-A)\left[(C-A) d^{2}+D-B d\right]}{A^{2}}+\frac{a_{1}[B-2 d(C-A)]^{2}}{A^{2}}\right\} \\
& -2 k l\left(3 a_{1}^{2} b_{1}+3 a_{0}^{2} a_{1}\right)-\rho a_{1}=0 ; \\
& \left(d+\frac{G^{\prime}}{G}\right)^{0}: k m\left(l^{2}-k^{2}\right)\left\{\frac{a_{1}[B-2 d(C-A)]\left[(C-A) d^{2}+D-B d\right]}{A^{2}}\right. \\
& \left.+\frac{b_{1}(C-A)[B-2 d(C-A)]}{A^{2}}\right\}-2 k l\left(a_{0}^{3}+6 a_{0} a_{1} b_{1}\right)-\rho a_{0}=0 ; \\
& \left(d+\frac{G^{\prime}}{G}\right)^{-1}: k m\left(l^{2}-k^{2}\right)\left\{\frac{b_{1}[B-2 d(C-A)]^{2}}{A^{2}}+\frac{2 b_{1}(C-A)\left[d^{2}(C-A)+D-B d\right]}{A^{2}}\right\} \\
& -2 k l\left(3 a_{1} b_{1}^{2}+3 a_{0}^{2} b_{1}\right)-\rho b_{1}=0 ; \\
& \left(d+\frac{G^{\prime}}{G}\right)^{-2}: k m\left(l^{2}-k^{2}\right) \frac{3 b_{1}[B-2 d(C-A)]\left[(C-A) d^{2}+D-B d\right]}{A^{2}}-3 k l \cdot 3 a_{0} b_{1}^{2}=0 \\
& \left(d+\frac{G^{\prime}}{G}\right)^{-3}: k m\left(l^{2}-k^{2}\right) \frac{2 b_{1}\left[d^{2}(C-A)+D-B d\right]^{2}}{A^{2}}-2 k l b_{1}^{3}=0 .
\end{aligned}
$$

Solving this system of algebraic equations using the computer algebra software Maple, we get the following results:

Case 1. $b_{1}=0, a_{0}=\mp \frac{m \psi\left(l^{2}-k^{2}\right)(B+2 d \psi)}{2 l|A \psi|} \sqrt{\frac{l}{m\left(l^{2}-k^{2}\right)}}, a_{1}= \pm \sqrt{\frac{m\left(l^{2}-k^{2}\right)}{l}} \frac{|\psi|}{|A|}, \rho=-\frac{k m\left(l^{2}-k^{2}\right)\left(B^{2}+4 D \psi\right)}{2 A^{2}}$.

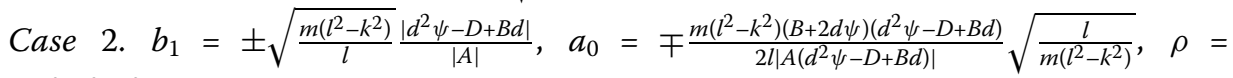
$-\frac{k m\left(l^{2}-k^{2}\right)\left(B^{2}+4 D \psi\right)}{2 A^{2}}$.

Substituting these solutions into (3.3), we obtain the traveling wave solutions as follows:

$$
\begin{aligned}
u(\xi)= & \mp \frac{m \psi\left(l^{2}-k^{2}\right)(B+2 d \psi)}{2 l|A \psi|} \sqrt{\frac{l}{m\left(l^{2}-k^{2}\right)}} \\
& \pm \sqrt{\frac{m\left(l^{2}-k^{2}\right)}{l} \frac{|\psi|}{|A|}\left(d+\frac{G^{\prime}(\xi)}{G(\xi)}\right),} \\
u(\xi)= & \mp \frac{m\left(l^{2}-k^{2}\right)(B+2 d \psi)\left(d^{2} \psi-D+B d\right)}{2 l\left|A\left(d^{2} \psi-D+B d\right)\right|} \sqrt{\frac{l}{m\left(l^{2}-k^{2}\right)}} \\
& \pm \sqrt{\frac{m\left(l^{2}-k^{2}\right)}{l} \frac{\left|d^{2} \psi-D+B d\right|}{|A|}\left(d+\frac{G^{\prime}(\xi)}{G(\xi)}\right)^{-1},}
\end{aligned}
$$

where $\frac{G^{\prime}(\xi)}{G(\xi)}$ is defined in Sect. $2, \xi=k x+m y+\frac{l t^{\alpha}}{\alpha}$. 


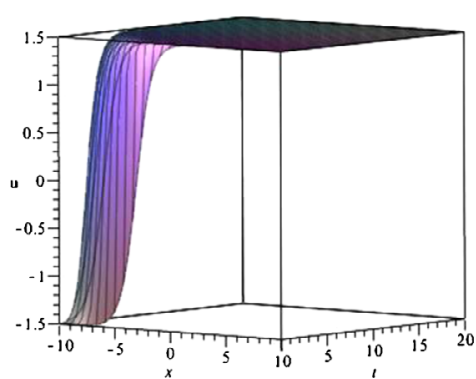

(a) $3 \mathrm{D}$ plot at $y=1$

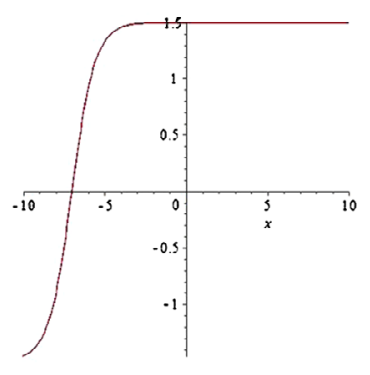

(b) $2 \mathrm{D}$ plot at $y=t=1$

Figure 1 Kink wave solution of equation (1.1) for $u_{1_{1}}(t, x, y)$ when $\alpha=\frac{1}{2}, A=B=l=2, C=D=k=1, m=3$

Family 1. When $B \neq 0, \psi=A-C$, and $\mu=B^{2}+4 D \psi>0$, we have the traveling wave solutions as follows:

$$
\begin{aligned}
& u_{1}(t, x, y) \\
& =\mp \frac{m \psi\left(l^{2}-k^{2}\right)(B+2 d \psi)}{2 l|A \psi|} \sqrt{\frac{l}{m\left(l^{2}-k^{2}\right)}} \pm \sqrt{\frac{m\left(l^{2}-k^{2}\right)}{l}} \frac{|\psi|}{|A|}\left[d+\frac{B}{2 \psi}\right. \\
& \left.+\frac{\sqrt{\mu}}{2 \psi} \frac{C_{1} \sinh \left(\frac{\sqrt{\mu}}{2 A}\left(k x+m y+\frac{l t^{\alpha}}{\alpha}\right)\right)+C_{2} \cosh \left(\frac{\sqrt{\mu}}{2 A}\left(k x+m y+\frac{l^{\alpha}}{\alpha}\right)\right)}{C_{1} \cosh \left(\frac{\sqrt{\mu}}{2 A}\left(k x+m y+\frac{l t^{\alpha}}{\alpha}\right)\right)+C_{2} \sinh \left(\frac{\sqrt{\mu}}{2 A}\left(k x+m y+\frac{l t^{\alpha}}{\alpha}\right)\right)}\right], \\
& u_{2}(t, x, y) \\
& =\mp \frac{m\left(l^{2}-k^{2}\right)(B+2 d \psi)\left(d^{2} \psi-D+B d\right)}{2 l\left|A\left(d^{2} \psi-D+B d\right)\right|} \sqrt{\frac{l}{m\left(l^{2}-k^{2}\right)}} \\
& \pm \sqrt{\frac{m\left(l^{2}-k^{2}\right)}{l}} \frac{\left|d^{2} \psi-D+B d\right|}{|A|}\left[d+\frac{B}{2 \psi}\right. \\
& \left.+\frac{\sqrt{\mu}}{2 \psi} \frac{C_{1} \sinh \left(\frac{\sqrt{\mu}}{2 A}\left(k x+m y+\frac{l t^{\alpha}}{\alpha}\right)\right)+C_{2} \cosh \left(\frac{\sqrt{\mu}}{2 A}\left(k x+m y+\frac{l t^{\alpha}}{\alpha}\right)\right)}{C_{1} \cosh \left(\frac{\sqrt{\mu}}{2 A}\left(k x+m y+\frac{l t^{\alpha}}{\alpha}\right)\right)+C_{2} \sinh \left(\frac{\sqrt{\mu}}{2 A}\left(k x+m y+\frac{l t^{\alpha}}{\alpha}\right)\right)}\right]^{-1},
\end{aligned}
$$

where $C_{1}$ and $C_{2}$ are arbitrary constants.

In particular, if $C_{1} \neq 0, \psi>0, l^{2}>k^{2}, m>0, A>0, l>0$, and $C_{2}=0$ in equation (3.6), we obtain the kink wave solutions

$$
u_{1_{1}}(t, x, y)=\frac{\sqrt{m\left(l^{2}-k^{2}\right)\left(B^{2}+4 D \psi\right)}}{2 A \sqrt{l}} \tanh \left(\frac{\sqrt{B^{2}+4 D \psi}}{2 A}\left(k x+m y+\frac{l t^{\alpha}}{\alpha}\right)\right) .
$$

Family 2. When $B \neq 0, \psi=A-C$, and $\mu=B^{2}+4 D \psi<0$, we obtain the following exact solutions:

$$
\begin{aligned}
u_{3}(t, x, y) & \\
=\mp & \frac{m \psi\left(l^{2}-k^{2}\right)(B+2 d \psi)}{2 l|A \psi|} \sqrt{\frac{l}{m\left(l^{2}-k^{2}\right)}} \pm \sqrt{\frac{m\left(l^{2}-k^{2}\right)}{l}} \frac{|\psi|}{|A|}\left[d+\frac{B}{2 \psi}\right. \\
& \left.+\frac{\sqrt{-\mu}}{2 \psi} \frac{-C_{1} \sin \left(\frac{\sqrt{-\mu}}{2 A}\left(k x+m y+\frac{l t^{\alpha}}{\alpha}\right)\right)+C_{2} \cos \left(\frac{\sqrt{-\mu}}{2 A}\left(k x+m y+\frac{l t^{\alpha}}{\alpha}\right)\right)}{C_{1} \cos \left(\frac{\sqrt{-\mu}}{2 A}\left(k x+m y+\frac{l t^{\alpha}}{\alpha}\right)\right)+C_{2} \sin \left(\frac{\sqrt{-\mu}}{2 A}\left(k x+m y+\frac{l t^{\alpha}}{\alpha}\right)\right)}\right] .
\end{aligned}
$$




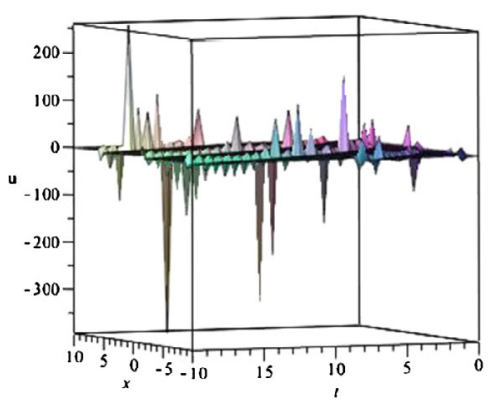

(a) $3 \mathrm{D}$ plot at $y=1$

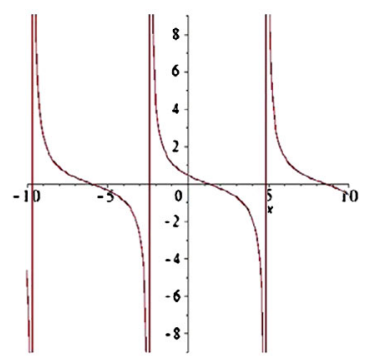

(b) 2D plot at $y=t=1$

Figure 2 Periodic wave solution of equation (1.1) for $u_{3_{1}}(t, x, y)$ when $\alpha=\frac{1}{2}, l=A=m=2, k=B=C=1$, $D=-1$

$$
\begin{aligned}
& u_{4}(t, x, y) \\
& =\mp \frac{m\left(l^{2}-k^{2}\right)(B+2 d \psi)\left(d^{2} \psi-D+B d\right)}{2 l\left|A\left(d^{2} \psi-D+B d\right)\right|} \sqrt{\frac{l}{m\left(l^{2}-k^{2}\right)}} \\
& \quad \pm \sqrt{\frac{m\left(l^{2}-k^{2}\right)}{l} \frac{\left|d^{2} \psi-D+B d\right|}{|A|}\left[d+\frac{B}{2 \psi}\right.} \\
& \left.\quad+\frac{\sqrt{-\mu}}{2 \psi} \frac{C_{1} \sin \left(\frac{\sqrt{-\mu}}{2 A}\left(k x+m y+\frac{l t^{\alpha}}{\alpha}\right)\right)+C_{2} \cos \left(\frac{\sqrt{-\mu}}{2 A}\left(k x+m y+\frac{l t^{\alpha}}{\alpha}\right)\right)}{C_{1} \cos \left(\frac{\sqrt{-\mu}}{2 A}\left(k x+m y+\frac{l t^{\alpha}}{\alpha}\right)\right)+C_{2} \sin \left(\frac{\sqrt{-\mu}}{2 A}\left(k x+m y+\frac{l t^{\alpha}}{\alpha}\right)\right)}\right]^{-1} .
\end{aligned}
$$

In particular, if $C_{1} \neq 0, \psi>0, l^{2}>k^{2}, m>0, A>0, l>0$, and $C_{2}=0$ in equation (3.9), we obtain the periodic wave solutions

$$
u_{3_{1}}(t, x, y)=-\frac{\sqrt{-m\left(l^{2}-k^{2}\right)\left(B^{2}+4 D \psi\right)}}{2 A \sqrt{l}} \tan \left(\frac{\sqrt{-\left(B^{2}+4 D \psi\right)}}{2 A}\left(k x+m y+\frac{l t^{\alpha}}{\alpha}\right)\right) .
$$

Family 3. When $B \neq 0, \psi=A-C$, and $\mu=B^{2}+4 \psi D=0$, we obtain

$$
\begin{aligned}
& u_{5}(t, x, y) \\
& =\mp \frac{m \psi\left(l^{2}-k^{2}\right)(B+2 d \psi)}{2 l|A \psi|} \sqrt{\frac{l}{m\left(l^{2}-k^{2}\right)}} \\
& \quad \pm \sqrt{\frac{m\left(l^{2}-k^{2}\right)}{l} \frac{|\psi|}{|A|}\left[d+\frac{B}{2 \psi}+\frac{C_{2}}{C_{1}+C_{2}\left(k x+m y+\frac{l t^{\alpha}}{\alpha}\right)}\right],} \\
& u_{6}(t, x, y) \\
& =\mp \frac{m\left(l^{2}-k^{2}\right)(B+2 d \psi)\left(d^{2} \psi-D+B d\right)}{2 l\left|A\left(d^{2} \psi-D+B d\right)\right|} \sqrt{\frac{l}{m\left(l^{2}-k^{2}\right)}} \\
& \quad \pm \sqrt{\frac{m\left(l^{2}-k^{2}\right)}{l}} \frac{\left|d^{2} \psi-D+B d\right|}{|A|}\left[d+\frac{B}{2 \psi}+\frac{C_{2}}{C_{1}+C_{2}\left(k x+m y+\frac{l t^{\alpha}}{\alpha}\right)}\right]^{-1} .
\end{aligned}
$$


Family 4. When $B=0, \psi=A-C$, and $\Delta=\psi D>0$, we obtain following traveling wave solutions:

$$
\begin{aligned}
& u_{7}(t, x, y) \\
& =\mp \frac{m \psi\left(l^{2}-k^{2}\right)(B+2 d \psi)}{2 l|A \psi|} \sqrt{\frac{l}{m\left(l^{2}-k^{2}\right)}} \pm \sqrt{\frac{m\left(l^{2}-k^{2}\right)}{l}} \frac{|\psi|}{|A|}[d \\
& \left.+\frac{\sqrt{\Delta}}{\psi} \frac{C_{1} \sinh \left(\frac{\sqrt{\Delta}}{A}\left(k x+m y+\frac{l t^{\alpha}}{\alpha}\right)\right)+C_{2} \cosh \left(\frac{\sqrt{\Delta}}{A}\left(k x+m y+\frac{l t^{\alpha}}{\alpha}\right)\right)}{C_{1} \cosh \left(\frac{\sqrt{\Delta}}{A}\left(k x+m y+\frac{l t^{\alpha}}{\alpha}\right)\right)+C_{2} \sinh \left(\frac{\sqrt{\Delta}}{A}\left(k x+m y+\frac{l t^{\alpha}}{\alpha}\right)\right)}\right], \\
& u_{8}(t, x, y) \\
& =\mp \frac{m\left(l^{2}-k^{2}\right)(B+2 d \psi)\left(d^{2} \psi-D+B d\right)}{2 l\left|A\left(d^{2} \psi-D+B d\right)\right|} \sqrt{\frac{l}{m\left(l^{2}-k^{2}\right)}} \\
& \pm \sqrt{\frac{m\left(l^{2}-k^{2}\right)}{l}} \frac{\left|d^{2} \psi-D+B d\right|}{|A|}[d \\
& \left.+\frac{\sqrt{\Delta}}{\psi} \frac{C_{1} \sinh \left(\frac{\sqrt{\Delta}}{A}\left(k x+m y+\frac{l t^{\alpha}}{\alpha}\right)\right)+C_{2} \cosh \left(\frac{\sqrt{\Delta}}{A}\left(k x+m y+\frac{l t^{\alpha}}{\alpha}\right)\right)}{C_{1} \cosh \left(\frac{\sqrt{\Delta}}{A}\left(k x+m y+\frac{l t^{\alpha}}{\alpha}\right)\right)+C_{2} \sinh \left(\frac{\sqrt{\Delta}}{A}\left(k x+m y+\frac{l t^{\alpha}}{\alpha}\right)\right)}\right]^{-1} .
\end{aligned}
$$

Family 5. When $B=0, \psi=A-C$, and $\Delta=\psi D<0$, we have

$$
\begin{aligned}
u_{9}(t, x, y) & \mp \frac{m \psi\left(l^{2}-k^{2}\right)(B+2 d \psi)}{2 l|A \psi|} \sqrt{\frac{l}{m\left(l^{2}-k^{2}\right)}} \pm \sqrt{\frac{m\left(l^{2}-k^{2}\right)}{l}} \frac{|\psi|}{|A|}[d \\
& \left.+\frac{\sqrt{-\Delta}}{\psi} \frac{-C_{1} \sin \left(\frac{\sqrt{-\Delta}}{A}\left(k x+m y+\frac{l t^{\alpha}}{\alpha}\right)\right)+C_{2} \cos \left(\frac{\sqrt{-\Delta}}{A}\left(k x+m y+\frac{l t^{\alpha}}{\alpha}\right)\right)}{C_{1} \cos \left(\frac{\sqrt{-\Delta}}{A}\left(k x+m y+\frac{l t^{\alpha}}{\alpha}\right)\right)+C_{2} \sin \left(\frac{\sqrt{-\Delta}}{A}\left(k x+m y+\frac{l t^{\alpha}}{\alpha}\right)\right)}\right] \\
u_{10}( & t, x, y) \\
= & \frac{m\left(l^{2}-k^{2}\right)(B+2 d \psi)\left(d^{2} \psi-D+B d\right)}{2 l\left|A\left(d^{2} \psi-D+B d\right)\right|} \sqrt{\frac{l}{m\left(l^{2}-k^{2}\right)}} \\
& \pm \sqrt{\frac{m\left(l^{2}-k^{2}\right)}{l} \frac{\left|d^{2} \psi-D+B d\right|}{l}[d}\left[\begin{array}{ll}
|A| \\
\end{array}\right. \\
& \left.+\frac{\sqrt{-\Delta}-C_{1} \sin \left(\frac{\sqrt{-\Delta}}{A}\left(k x+m y+\frac{l t^{\alpha}}{\alpha}\right)\right)+C_{2} \cos \left(\frac{\sqrt{-\Delta}}{A}\left(k x+m y+\frac{l t^{\alpha}}{\alpha}\right)\right)}{C_{1} \cos \left(\frac{\sqrt{-\Delta}}{A}\left(k x+m y+\frac{l t^{\alpha}}{\alpha}\right)\right)+C_{2} \sin \left(\frac{\sqrt{-\Delta}}{A}\left(k x+m y+\frac{l t^{\alpha}}{\alpha}\right)\right)}\right]^{-1} .
\end{aligned}
$$

\subsection{Bifurcation, phase portraits, and exact solutions for equation (1.1) using the bifurcation method}

Let $\frac{d u}{d \xi}=y$. Then, equation (3.2) is equivalent to the following 2-dimensional system:

$$
\left\{\begin{array}{l}
\frac{d u}{d \xi}=y, \\
\frac{d y}{d \xi}=-\beta u^{3}-\gamma u,
\end{array}\right.
$$




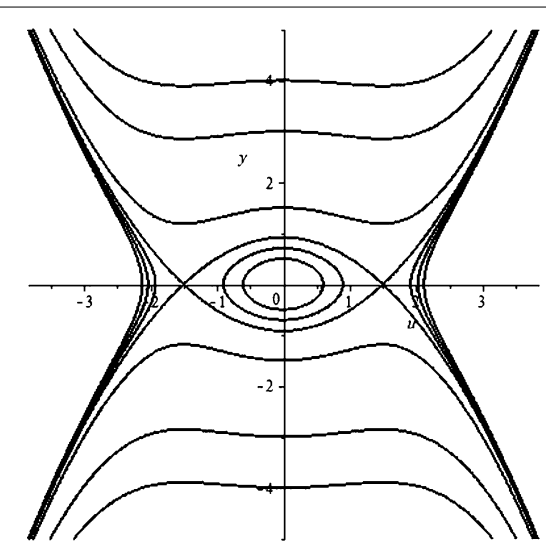

(a) $\beta<0, \gamma>0$

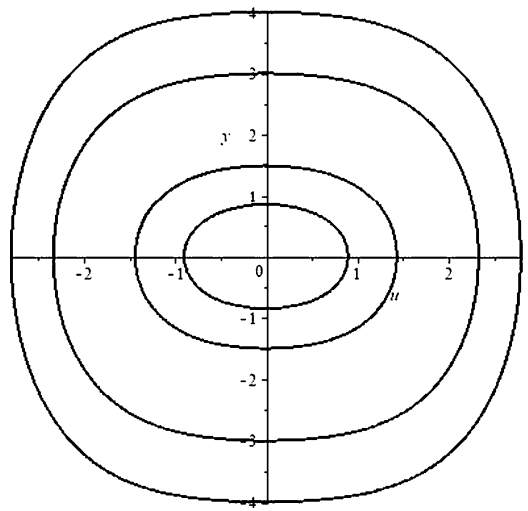

(c) $\beta>0, \gamma>0$

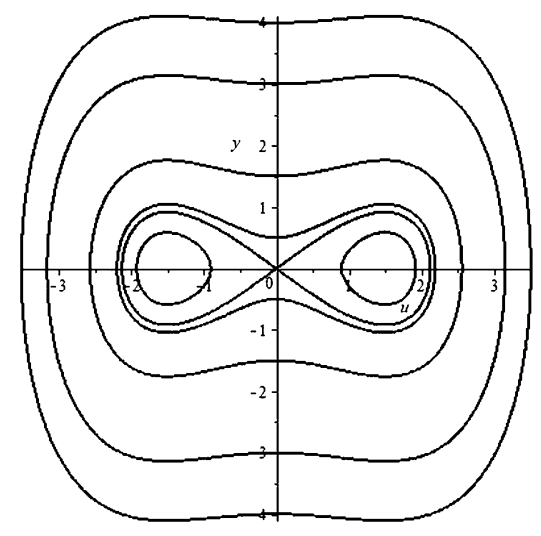

(b) $\beta>0, \gamma<0$

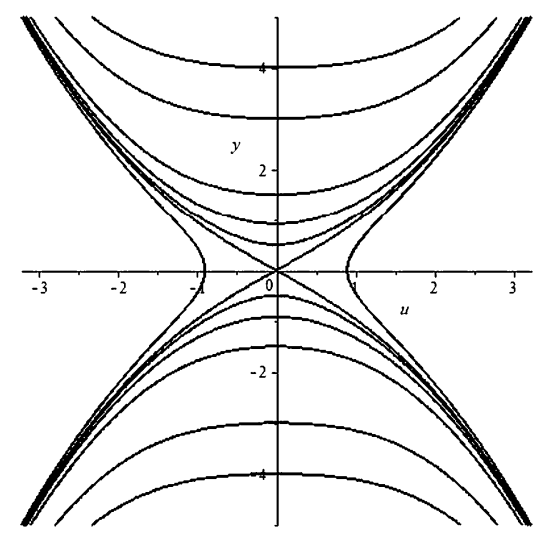

(d) $\beta<0, \gamma<0$

Figure 3 The phase portraits of system (3.18) for $\beta \gamma \neq 0$

which has the first integral

$$
H(u, y)=\frac{1}{2} y^{2}+\frac{\beta}{4} u^{4}+\frac{\gamma}{2} u^{2}=h
$$

where $\beta=\frac{2 l}{m\left(k^{2}-l^{2}\right)}$ and $\gamma=\frac{\rho}{k m\left(k^{2}-l^{2}\right)}$.

Let $f(u)=-\beta u^{3}-\gamma u$. If $\beta \gamma<0$, we obtain three zeros of $f(u): u_{1}=-\sqrt{-\frac{\gamma}{\beta}}, u_{2}=0$, and $u_{3}=\sqrt{-\frac{\gamma}{\beta}}$. If $\beta \gamma>0$, we obtain one zero of $f(u)$, namely $u_{4}=0$. We assume that $P_{i}\left(u_{i}, 0\right)(i=$ $1,2,3)$ is the equilibrium points of system (3.18). Clearly, the eigenvalue of system (3.18) at this point is $\lambda_{1,2}=\sqrt{f^{\prime}(u)}$. By the bifurcation theory, we derive the phase portraits of system (3.18) shown in Fig. 3.

I. The case $\beta<0, \gamma>0$.

In this case, there exist three equilibrium points of system (3.18), where $P_{1}\left(-\sqrt{-\frac{\gamma}{\beta}}, 0\right)$ and $P_{3}\left(\sqrt{-\frac{\gamma}{\beta}}, 0\right)$ are saddle points, while $P_{2}(0,0)$ is a center. Two heteroclinic orbits connect $P_{1}\left(-\sqrt{-\frac{\gamma}{\beta}}, 0\right)$ and $P_{3}\left(\sqrt{-\frac{\gamma}{\beta}}, 0\right)$; moreover, a family of periodic orbits enclose $P_{2}(0,0)$ in Fig. 3(a) defined by equation (3.19). 
(i) Suppose that $h \in\left(0,-\frac{\gamma^{2}}{4 \beta}\right)$. Then a family of periodic orbits of system (3.18) are defined by the algebraic equation

$$
y= \pm \sqrt{-\frac{\beta}{2}} \sqrt{u^{4}+\frac{2 \gamma}{\beta} u^{2}-\frac{4 h}{\beta}}= \pm \sqrt{-\frac{\beta}{2}} \sqrt{\left(\phi_{1 h}^{2}-u^{2}\right)\left(\phi_{2 h}^{2}-u^{2}\right)},
$$

where $\phi_{1 h}=\sqrt{-\frac{\gamma}{\beta}-\frac{1}{\beta} \sqrt{\gamma^{2}+4 \beta h}}, \phi_{2 h}=\sqrt{-\frac{\gamma}{\beta}+\frac{1}{\beta} \sqrt{\gamma^{2}+4 \beta h}}$.

By employing (3.20) and the first equation of (3.18), we integrate them along the periodic orbits and obtain

$$
\int_{0}^{u} \frac{d \varphi}{\sqrt{\left(\phi_{1 h}^{2}-\varphi^{2}\right)\left(\phi_{2 h}^{2}-\varphi^{2}\right)}}= \pm \sqrt{-\frac{\beta}{2}}\left(\xi-\xi_{0}\right) .
$$

Hence, we can obtain two families of periodic traveling wave solutions, namely

$$
u_{1}(t, x, y)= \pm \phi_{1 h} \operatorname{sn}\left(\phi_{2 h} \sqrt{-\frac{\beta}{2}}\left(k x+m y+\frac{l t^{\alpha}}{\alpha}-\xi_{0}\right), \frac{\phi_{1 h}}{\phi_{2 h}}\right) .
$$

Remark 3.1 In (3.22), $u_{1}(t, x, y)$ represents Jacobi elliptic function solutions. When $\frac{\phi_{1 h}}{\phi_{2 h}} \rightarrow$ $0, \operatorname{sn}\left(\phi_{2 h} \sqrt{-\frac{\beta}{2}}\left(k x+m y+\frac{l t^{\alpha}}{\alpha}-\xi_{0}\right), \frac{\phi_{1 h}}{\phi_{2 h}}\right)=\sin \left(\phi_{2 h} \sqrt{-\frac{\beta}{2}}\left(k x+m y+\frac{l t^{\alpha}}{\alpha}-\xi_{0}\right)\right)$. When $\frac{\phi_{1 h}}{\phi_{2 h}} \rightarrow 1$, $\operatorname{sn}\left(\phi_{2 h} \sqrt{-\frac{\beta}{2}}\left(k x+m y+\frac{l t^{\alpha}}{\alpha}-\xi_{0}\right), \frac{\phi_{1 h}}{\phi_{2 h}}\right)=\tanh \left(\phi_{2 h} \sqrt{-\frac{\beta}{2}}\left(k x+m y+\frac{l t^{\alpha}}{\alpha}-\xi_{0}\right)\right)$.

(ii) Suppose that $h=-\frac{\gamma^{2}}{4 \beta}$. Then we have $\phi_{1 h}=\phi_{2 h}=\sqrt{-\frac{\gamma}{\beta}}$ and obtain two families of kink solitary wave solutions,

$$
u_{2}(t, x, y)= \pm \sqrt{-\frac{\gamma}{\beta}} \tanh \left(\sqrt{\frac{\gamma}{2}}\left(k x+m y+\frac{l t^{\alpha}}{\alpha}-\xi_{0}\right)\right) .
$$

II. The case $\beta>0, \gamma<0$.

In this case, $P_{1}\left(-\sqrt{-\frac{\gamma}{\beta}}, 0\right)$ and $P_{3}\left(\sqrt{-\frac{\gamma}{\beta}}, 0\right)$ are center points, while $P_{2}(0,0)$ is a saddle point.

(i) Suppose that $h \in\left(-\frac{\gamma^{2}}{4 \beta}, 0\right)$. Two families of periodic orbits of system (3.18) are defined by the algebraic equation

$$
\begin{aligned}
y & = \pm \sqrt{\frac{\beta}{2}} \sqrt{-u^{4}-\frac{2 \gamma}{\beta} u^{2}+\frac{4 h}{\beta}} \\
& = \pm \sqrt{\frac{\beta}{4}} \sqrt{\left(u^{2}-\chi_{1 h}^{2}\right)\left(\chi_{2 h}^{2}-u^{2}\right)}
\end{aligned}
$$

where $\chi_{1 h}=\sqrt{-\frac{\gamma}{\beta}-\frac{1}{\beta} \sqrt{\gamma^{2}+4 \beta h}}, \chi_{2 h}=\sqrt{-\frac{\gamma}{\beta}+\frac{1}{\beta} \sqrt{\gamma^{2}+4 \beta h}}$.

Integrating them along the periodic orbits, we obtain two families of periodic traveling wave solutions, namely

$$
u_{3}(t, x, y)= \pm \chi_{2 h} \operatorname{dn}\left(\chi_{2 h} \sqrt{\frac{\beta}{2}}\left(k x+m y+\frac{l t^{\alpha}}{\alpha}-\xi_{0}\right), \frac{\sqrt{\chi_{2 h}^{2}-\chi_{1 h}^{2}}}{\chi_{2 h}}\right) .
$$


(ii) Suppose that $h=0$. Then we obtain two bell solitary wave solutions

$$
u_{4}(t, x, y)= \pm \sqrt{-\frac{2 \gamma}{\beta}} \operatorname{sech}\left(\sqrt{-\gamma}\left(k x+m y+\frac{l t^{\alpha}}{\alpha}-\xi_{0}\right)\right)
$$

(iii) Suppose that $h \in(0,+\infty)$. Then the function (3.19) can be rewritten as the following algebraic expression:

$$
y= \pm \sqrt{\frac{\beta}{2}} \sqrt{-u^{4}-\frac{2 \gamma}{\beta} u^{2}+\frac{4 h}{\beta}}= \pm \sqrt{\frac{\beta}{2}} \sqrt{\left(\chi_{3 h}^{2}+u^{2}\right)\left(\chi_{4 h}^{2}-u^{2}\right)}
$$

where $\chi_{3 h}=\sqrt{\frac{\gamma}{\beta}+\frac{1}{\beta} \sqrt{\gamma^{2}+4 \beta h}}, \chi_{4 h}=\sqrt{-\frac{\gamma}{\beta}+\frac{1}{\beta} \sqrt{\gamma^{2}+4 \beta h}}$.

Integrating them along the periodic orbits, we obtain two families of periodic traveling wave solutions, namely

$$
u_{5}(t, x, y)=\chi_{4 h} \operatorname{cn}\left(\sqrt{\frac{\beta\left(\chi_{3 h}^{2}+\chi_{4 h}^{2}\right)}{2}\left(k x+m y+\frac{l t^{\alpha}}{\alpha}-\xi_{0}\right)}, \frac{\chi_{4 h}}{\sqrt{\chi_{3 h}^{2}+\chi_{4 h}^{2}}}\right) .
$$

\section{Conclusion}

By using the generalized $\left(G^{\prime} / G\right)$-expansion method and bifurcation theory method, we obtain exact traveling wave solutions, bifurcation, and phase portraits for the $(2+1)$ dimensional conformable time-fractional Zoomeron equation under the given parameter conditions. Many exact solutions have been obtained, which include hyperbolic function solutions, Jacobi elliptic function solutions, trigonometric function solutions, and rational function solutions. Compared with the previous work, the solution method obtained in the paper has not been reported. Furthermore, two methods we employ here can be used to analyze the exact solutions and bifurcation for other FPDE.

\section{Appendix}

In [35], Atangana et al. have proved some important properties of the conformable fractional derivative. Next, we give the proof details, which are taken from [35].

Proof To prove Remark 2.1(i), let $\varepsilon=t^{\alpha-1} h$ in Definition 2.1. Then,

$$
\begin{aligned}
D_{t}^{\alpha} f(t) & =\lim _{\varepsilon \rightarrow 0} \frac{f\left(t+\varepsilon t^{1-\alpha}\right)-f(t)}{\varepsilon}=\lim _{h \rightarrow 0} \frac{f(t+h)-f(t)}{h t^{\alpha-1}} \\
& =t^{1-\alpha} \lim _{h \rightarrow 0} \frac{f(t+h)-f(t)}{h}=t^{1-\alpha} \frac{d f}{d t}(t) ;
\end{aligned}
$$

when $f(t)=t^{\mu}, \mu \in \mathbf{R}$, we obtain

$$
D_{t}^{\alpha} t^{\mu}=t^{1-\alpha} \frac{d t^{\mu}}{d t}=t^{1-\alpha} \mu t^{\mu-1}=\mu t^{\mu-\alpha} .
$$

To prove Remark 2.1(iii), by Definition 2.1, we have the following:

$$
D_{t}^{\alpha}(f \circ g)(t)=\lim _{\varepsilon \rightarrow 0} \frac{(f \circ g)\left(t+\varepsilon t^{1-\alpha}\right)-(f \circ g)(t)}{\varepsilon} .
$$


Now putting $k=\varepsilon t^{1-\alpha}$, we obtain

$$
D_{t}^{\alpha}(f \circ g)(t)=t^{1-\alpha} \lim _{k \rightarrow 0} \frac{(f \circ g)(t+k)-(f \circ g)(t)}{k},
$$

with

$$
\lim _{k \rightarrow 0} \frac{(f \circ g)(t+k)-(f \circ g)(t)}{k} .
$$

So we obtain

$$
\lim _{k \rightarrow 0} \frac{(f \circ g)(t+k)-(f \circ g)(t)}{k}=\lim _{k \rightarrow 0} \frac{f(g(t)+k)-f(g(t))}{k},
$$

If $k=\varepsilon(g(t))^{1-\alpha}$, then formula (A.3) can be transformed to

$$
\lim _{k \rightarrow 0} \frac{(f \circ g)(t+k)-(f \circ g)(t)}{k}=(g(t))^{\alpha-1} \lim _{\varepsilon \rightarrow 0} \frac{f\left(g(t)+\varepsilon(g(t))^{1-\alpha}\right)-f(g(t))}{\varepsilon} .
$$

Now replacing (A.4) in (A.2), we obtain the following:

$$
D_{t}^{\alpha}(f \circ g)(t)=t^{1-\alpha} g(t)^{\alpha-1} \lim _{\varepsilon \rightarrow 0} \frac{f\left(g(t)+\varepsilon(g(t))^{1-\alpha}\right)-f(g(t))}{\varepsilon},
$$

with, of course, yields

$$
\left.D_{t}^{\alpha}(f(t))\right|_{t=g(t)}=\lim _{\varepsilon \rightarrow 0} \frac{f\left(g(t)+\varepsilon(g(t))^{1-\alpha}\right)-f(g(t))}{\varepsilon} .
$$

\section{Acknowledgements}

The authors would like to thank the reviewers for their constructive suggestions and helpful comments.

\section{Funding}

This work was supported by Scientific Research Funds of Chengdu University under grant No. 2081920034.

Availability of data and materials

Not applicable.

\section{Competing interests}

The authors declare that they have no competing interests.

\section{Authors' contributions}

Both authors read and approved the final manuscript.

\section{Publisher's Note}

Springer Nature remains neutral with regard to jurisdictional claims in published maps and institutional affiliations.

Received: 10 June 2020 Accepted: 12 November 2020 Published online: 23 November 2020

\section{References}

1. Kaur, J., Gupta, R.K., Kumar, S.: On explicit exact solutions and conservation laws for time fractional variable-coefficient coupled Burger's equations. Commun. Nonlinear Sci. Numer. Simul. 83, 1-24 (2020)

2. Zhang, Z.Y., Li, G.F.: Lie symmetry analysis and exact solutions of the time-fractional biological population model. Phys. A, Stat. Mech. Appl. 540, 1-11 (2020)

3. Baleanu, D., Inc, M., Yusuf, A., Aliyu, A.I.: Lie symmetry analysis, exact solutions and conservation laws for the time fractional Caudrey-Dodd-Gibbon-Sawada-Kotera equation. Commun. Nonlinear Sci. Numer. Simul. 59, 222-234 (2018)

4. Korkmaz, A.: Exact solutions of space-time fractional EW and modified EW equations. Chaos Solitons Fractals 96 , 132-138 (2017) 
5. Rui, W.G.: Applications of homogenous balanced principle on investigating exact solutions to a series of time fractional nonlinear PDEs. Commun. Nonlinear Sci. Numer. Simul. 47, 253-266 (2017)

6. Alquran, M., Jaradat, H.M., Syam, M.I.: Analytical solution of the time-fractional Phi-4 equation by using modified residual power series method. Nonlinear Dyn. 4, 2525-2529 (2017)

7. Wu, C., Rui, W.G.: Method of separation variables combined with homogenous balanced principle for searching exact solutions of nonlinear time-fractional biological population model. Commun. Nonlinear Sci. Numer. Simul. 63. 88-100 (2018)

8. Du, L.X., Sun, Y.H., Wu, D.S.: Bifurcations and solutions for the generalized nonlinear Schrödinger equation. Phys. Lett. A 383, 126028-126033 (2019)

9. Rui, W.G.: Applications of integral bifurcation method together with homogeneous balanced principle on investigating exact solutions of time fractional nonlinear PDEs. Nonlinear Dyn. 91, 697-712 (2018)

10. Ray, S.S.: Analytical solution for the space fractional diffusion equation by two-step Adomian decomposition method Commun. Nonlinear Sci. Numer. Simul. 41, 1295-1306 (2009)

11. Sahadevan, R., Prakash, P.: Exact solution of certain time fractional nonlinear partial differential equations. Nonlinear Dyn. 86, 1-15 (2016)

12. Baleanu, D., Mohammadi, H., Rezapour, S.: Analysis of the model of HIV-1 infection of $C D 4^{+} \mathrm{T}$-cell with a new approach of fractional derivative. Adv. Differ. Equ. 2020, 71 (2020)

13. Baleanu, D., Jajarmi, A., Mohammadi, H., Rezapour, S.: A new study on the mathematical modelling of human liver with Caputo-Fabrizio fractional derivative. Chaos Solitons Fractals 134, 1-7 (2020)

14. Baleanu, D., Mohammadi, H., Rezapour, S.: A mathematical theoretical study of a particular system of Caputo-Fabrizio fractional differential equations for Rubella disease model. Adv. Differ. Equ. 2020, 184 (2020)

15. Baleanu, D., Rezapour, S., Mohamadi, H.: Some existence results on nonlinear fractional differential equations. Philos. Trans. R. Soc. A, Math. Phys. Eng. Sci. 1990, 1-7 (2013)

16. Baleanu, D., Etemad, S., Rezapour, S.: A hybrid Caputo fractional modeling for thermostat with hybrid boundary value conditions. Bound. Value Probl. 2020, 1 (2020)

17. Aydogan, M.S., Baleanu, D., Mousalou, A., Etemad, S., Rezapour, S.: On high order fractional integro-differential equations including the Caputo-Fabrizio derivative. Bound. Value Probl. 2018, 1 (2018)

18. Ahmad, B., Alsaedi, A., Nazami, S.Z., Rezapour, S.: Some existence theorems for fractional integro-differential equations and inclusions with initial and non-separated boundary conditions. Bound. Value Probl. 2014, 1 (2014)

19. Baleanu, D., Agarwal, R.P., Mohammadi, H., Rezapour, S.: Some existence results for a nonlinear fractional differential equation on partially ordered Banach spaces. Bound. Value Probl. 2013, 1 (2013)

20. Wang, M.L., Li, X.Z., Zhang, J.L.: The $\left(\frac{G^{\prime}}{6}\right)$-expansion method and travelling wave solutions of nonlinear evolution equations in mathematical physics. Phys. Lett. A 372, 417-423 (2008)

21. Khalil, R., Horani, M.A., Yousef, A., Sababheh, M.: A new definition of fractional derivative. J. Comput. Appl. Math. 264 65-70 (2014)

22. Rezazadeh, H., Tariq, H., Eslami, M., Mohammad, M., Zhou, Q:: New exact solutions of nonlinear conformable time-fractional Phi-4 equation. Chin. J. Phys. 6, 2805-2816 (2018)

23. Shoukry, E.G., Mohammed, O.A.: New abundant wave solutions of the conformable space-time fractional $(4+1)$-dimensional Fokas equation in water waves. Comput. Math. Appl. 78, 2094-2106 (2019)

24. Thabet, H., Kendre, S.: Analytical solutions for conformable space-time fractional partial differential equations via fractional differential transform. Chaos Solitons Fractals 109, 238-245 (2018)

25. Kumar, D., Seadawy, A.R., Joardar, A.K.: Modified Kudryashov method via new exact solutions for some conformable fractional differential equations arising in mathematical biology. Chin. J. Phys. 56(1), 75-85 (2018)

26. Liu, Z.R., Li, J.B.: Bifurcation of solitary waves and domain wall waves for KdV-like equation with higher order nonlinearity. Int. J. Bifurc. Chaos 12, 397-407 (2002)

27. He, B., Meng, Q., Long, Y.: The bifurcation and exact peakons, solitary and periodic wave solutions for the Kudryashov-Sinelshchikov equation. Commun. Nonlinear Sci. Numer. Simul. 17, 4137-4148 (2012)

28. Liu, H.H., Yan, F.: Bifurcation and exact travelling wave solutions for Gardner-KP equation. Appl. Math. Comput. 228 384-394 (2012)

29. Zhang, B., Xia, Y.H., Zhu, W.J., Bai, Y.Z.: Explicit exact traveling wave solutions and bifurcations of the generalized combined double sinh-cosh-Gordon equation. Appl. Math. Comput. 363, 124576 (2019)

30. He, B.: Bifurcations and exact bounded travelling wave solutions for a partial differential equation. Nonlinear Anal., Real World Appl. 110, 364-371 (2010)

31. Odabasi, M.: Traveling wave solutions of conformable time-fractional Zakharov-Kuznetsov and Zoomeron equations Chin. J. Phys. 64, 194-202 (2020)

32. Kumar, D., Kaplan, M.: New analytical solutions of $(2+1)$-dimensional conformable time fractional Zoomeron equation via two distinct techniques. Chin. J. Phys. 53, 2173-2185 (2018)

33. Hosseini, K., Korkmaz, A., Sanadani, F., Zabihi, A., Topsakal, M.: New wave form solutions of nonlinear conformable time-fractional Zoomeron equation in (2 +1)-dimensions. Waves Random Complex Media 29, 1-11 (2019)

34. Raza, N.: Exact periodic and explicit solutions of the conformable time fractional Ginzburg-Landau equation. Opt. Quantum Electron. 50, 154-170 (2018)

35. Atangana, A., Baleanu, D., Alsaedi, A.: New properties of conformable derivative. Open Math. 13, 1-10 (2015) 